\title{
CHIARI MALFORMATION TYPE I - CASE REPORT AND REVIEW OF LITERATURE
}

\author{
Ioana Cociasu' ${ }^{1}$ Mihnea Pastia ${ }^{1}$, Irene Davidescu ${ }^{1,2}$, Ioan Buraga ${ }^{1,2}$, \\ Bogdan O. Popescu ${ }^{1,2,3}$ \\ ${ }^{1}$ Department of Neurology, Colentina Clinical Hospital, Bucharest, Romania \\ ${ }^{2}$ Department of Neurology, "Carol Davila" University of Medicine and Pharmacy, Bucharest, \\ Romania \\ ${ }^{3}$ Department of Molecular Medicine, "Victor Babes" National Institute of Pathology, Bucharest, \\ Romania
}

\begin{abstract}
Chiari malformations are congenital defects involving the cerebral structures in the posterior fossa. They range from asymptomatic cerebellar tonsil herniation beside the brainstem, to cerebellar aplasia. These conditions were first described in 1890 and are still considered a rare occurrence even though they are more likely under-diagnosed rather than rare. We report the case of a 44 year old woman with Chiari type I malformation and associated cervical syringomyelia.
\end{abstract}

Keywords: type I, syringomyelia, syrinx, Arnold-Chiari, headache, tonsil herniation, congenital

\section{INTRODUCTION}

Chiari malformations are congenital heterogeneous defects characterized by anomalies of the cerebellum, brainstem and occipito-cervical junction with varying degrees of downward displacement of the above-mentioned structures through the foramen magnum and into the cervical canal (1). These malformations are considered rare but advances in neuroimaging and their increasing availability has proven they are, in fact, not as rare as previously thought.

\section{CASE PRESENTATION}

We report the case of a 44-year-old woman that was admitted in our Neurology Department for suboccipital headaches. Her history is positive for smoking, duodenal ulcer and severe aortic stenosis for which she underwent surgical prosthesis placement 4 years previously. The headaches first appeared two years prior and have been present ever since with periods of improvement followed by worsening. The reason she came to the hospital was that the headaches had worsened over the previous month and had subsequently affected her capacity to carry out her day-to-day activities, including work. The pain is located mainly in the cervical area with extension over the occipital area bilaterally. She also describes slight memory impairment, fatigue and bouts of vertigo. She is the only person in her family to have this type of headache. She has tried taking medication including NSAIDs, tricyclic antidepressants and triptans without significant improvement.

During the general examination we found her to be overweight $(\mathrm{BMI}=28)$ with bilateral peripheral edema. Otherwise she is in good health.

The neurological examination finds both Arnold points to be painful upon applying pressure, but otherwise was unremarkable.

We performed a cervical spine radiograph that was normal.

Taking in consideration the patient complaints and the course of the pain, we decided to perform a head MRI. Sagittal $T_{1}$ - and $T_{2}$-weighted magnetic resonance imaging (MRI) obtained demonstrates peg-like cerebellar tonsils, which protrude through the foramen magnum over a length of $8 \mathrm{~mm}$ and 
associated syrinx at the C1-C3 level consistent with Chiari type I malformation with syringomyelia. Tonsil herniation does not appear to impede CSF flow (Fig. 1, 2, 3).

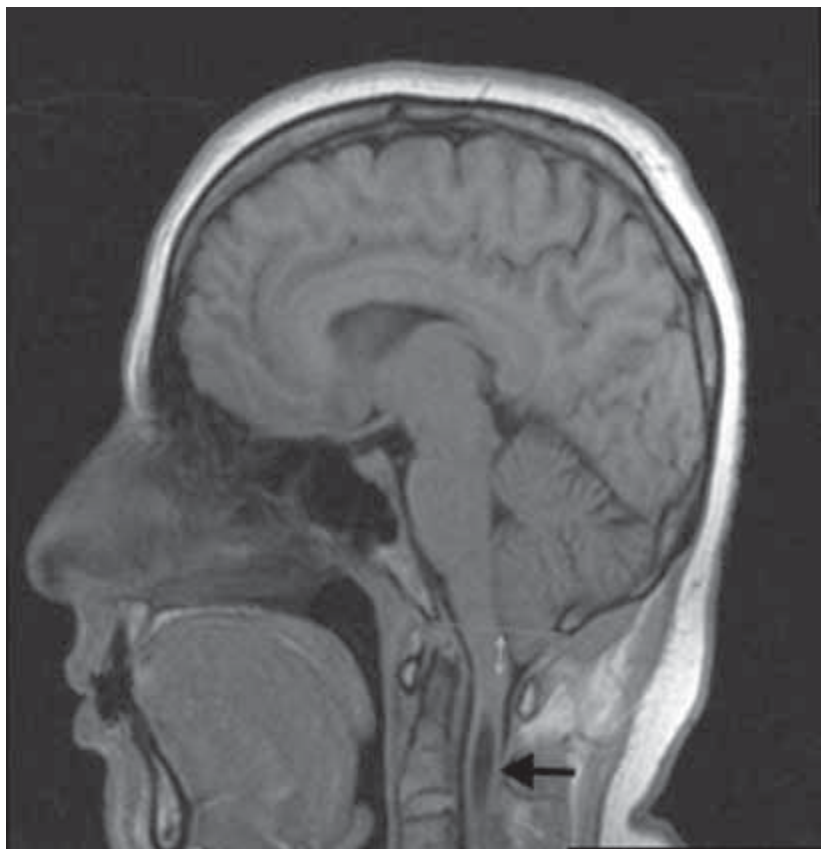

FIGURE 1. Sagittal T1-weighted image showing peg-like cerebellar tonsils, that herniate through foramen magnum $8 \mathrm{~mm}$ (green arrow) and associated syrinx (black arrow).

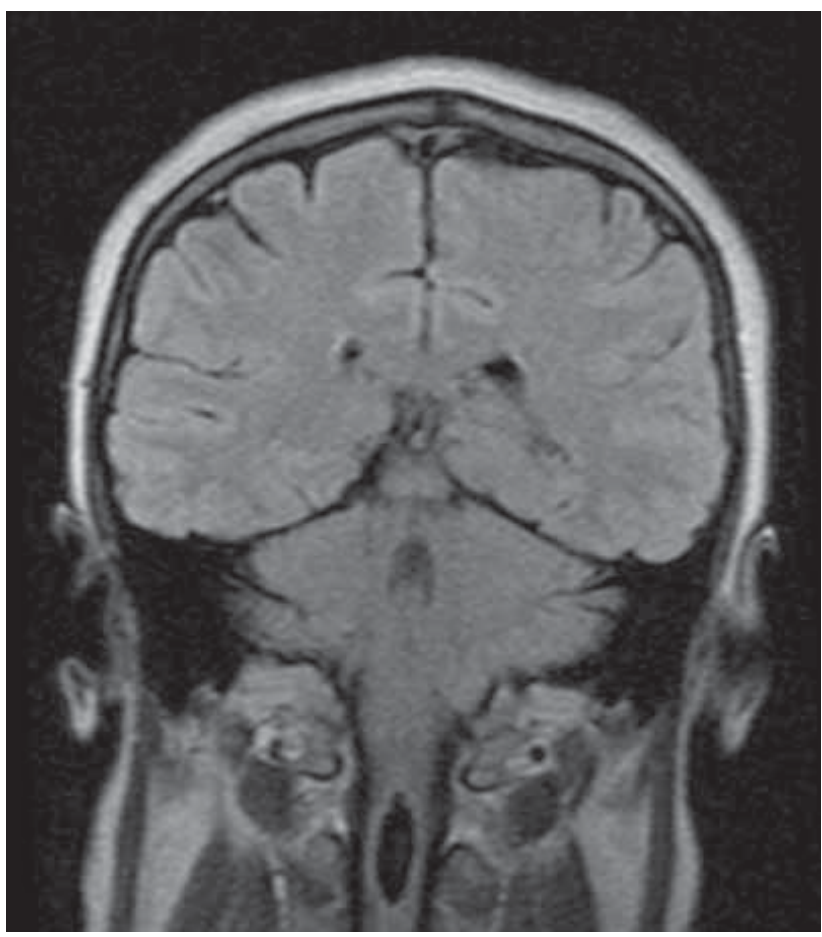

FIGURE 2. Coronal FLAIR image showing asymmetric cerebellar tonsil herniation through foramen magnum and associated syrinx.

After consulting with the patient, we decided to treat her conservatively with carbonic anhidrase in-

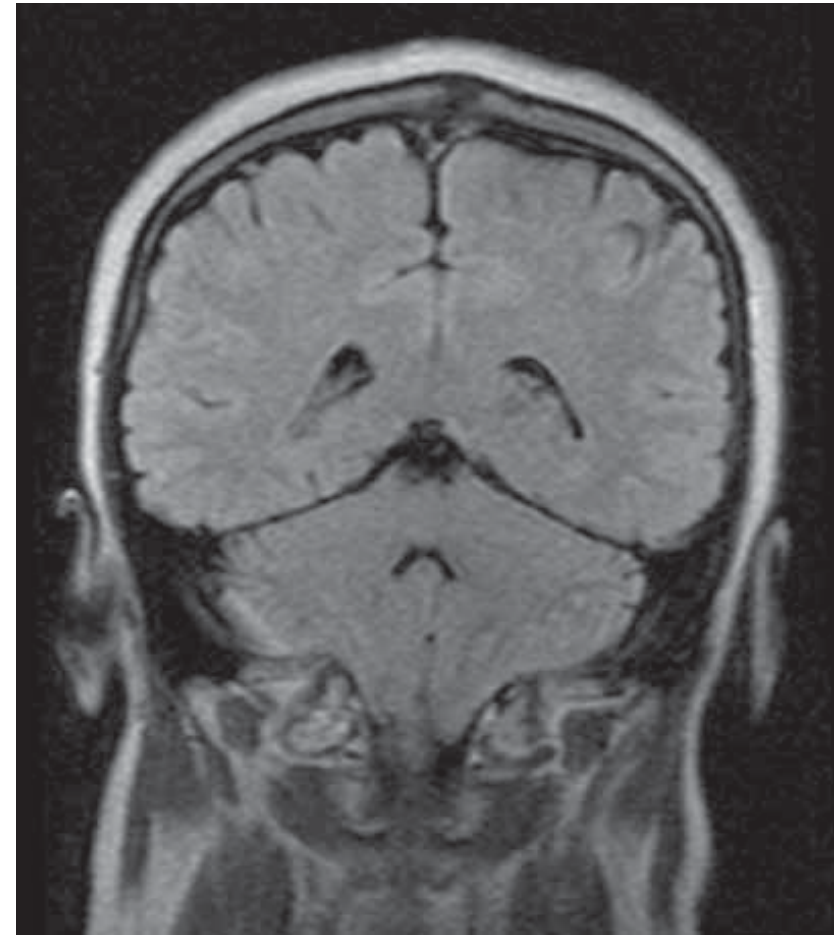

FIGURE 3. Coronal FLAIR image showing asymmetric cerebellar tonsil herniation (left > right) through foramen magnum.

hibitors and to monitor the Chiari malformation at 6 months. At present, the patient's headaches have improved significantly with the medication.

It was remarkable the fact that hypoesthesia due to the cavity was not present yet.

\section{DISCUSSION}

Austrian pathologist Hans Chiari makes the first complete description of these malformations in 1890 after observation on several autopsy specimens. Traditionally, Chiari described four degrees of severity all involving rombencephalic derivatives. A few years later, Julius Arnold published a case report of an infant with extended herniation of the brainstem and cerebellum through the foramen magnum, suggestive for what is now known as Chiari type II. The eponym now widely used (Arnold-Chiari malformation) was established in 1907 by a couple of Dr. Arnold's students (2).

Chiari malformations are classically classified into 4 types, from I to IV; the first three represent progressively severe displacement of the cerebellum and the brainstem through the foramen magnum and subsequent impairment of cerebrospinal fluid (CSF) flow. The most severe, type IV is used to describe cases in which cerebellar hypoplasia or aplasia is found. In time, this classification has been improved by the addition of subtypes - type 0 in which the patient has a small posterior fossa and a 
small, cone-like foramen magnum but without any displacement of the cerebellum and type 1.5 which is used for patients with characteristics pertaining to both type I and type II (3).

Chiari type I is characterized by inferior cerebellar migration through the foramen magnum (basion-opisthion line) and into the cervical canal of at least $5 \mathrm{~mm}$. It is frequently associated with syringomyelia. Concomitant hydrocephalus is rare.

Chiari type II, also known as Arnold-Chiari malformation, is characterized by downward migration of the cerebellum - tonsils and vermis and the forth ventricle; the brainstem appears misshapen. It is found almost always in the context of myelodysplasia and hydrocephalus. Syringomyelia is almost always found in these patients.

Chiari type III is the most rare of all Chiari malformations. It is characterized by a combination of a small posterior fossa with downward displacement of the cerebellum and the brainstem into a posterior encephalocele.

Chiari type IV is characterized by cerebellum hypoplasia or aplasia and is only historically still part of the Chiari malformations $(1,3,4)$.

Either one of the above mentioned anomalies can be accompanied by bony defects such as atlas assimilation, atlantoaxial dislocation, Klippel-Feil anomaly, platybasia, basilar invagination or lacunar skull (1).

Chiari type I is the most frequently diagnosed abnormality of all Chiari malformations. A small hypoplastic posterior fossa, in situ crowding and compression of the neural tissue and forced transforaminal displacement of the cerebellar tonsils define it. This definition, while anatomically precise can be misleading considering the fact that tonsillar herniation can be attributed to intracranial processes that are not malformations such as hydrocephalus, intracranial space occupying lesions, prolonged lumbo-peritoneal shunting, CSF leaks, tethered cord syndrome, craniosynostosis, acromegaly, and Paget's disease.

Milhorat et al performed posterior cranial fossa morphometric measurements on 752 patients with Chiari malformations and concluded that the size of the bony elements (posterior fossa volume, occipital bone and foramen magnum size) is diminished in Chiari malformations as opposed to hydrocephalus, intracranial mass lesions, and occipitoatlantoaxial joint instability or prolonged lumboperitoneal shunting $(5,6)$.

Chiari malformations are rare diseases, but some forms are more frequently encountered, especially since access to modern imaging techniques has in- creased in the last decade. The latest reported prevalence rates range between $0.1-0.5 \%$ with a slight female predominance. Chiari type I malformation is the most frequent across all age groups, and Chiari type II is the most frequent in pediatric populations $(4,7)$.

Patients with Chiari type I can be asymptomatic or minimally symptomatic. Asymptomatic patients are diagnosed after incidental discovery on imaging studies performed for matters unrelated to Chiari defects $(8,9)$.

Symptomatic patients present symptoms that pertain to elevated intracranial pressure, cranial neuropathies, medullary compression, myelopathy, cerebellar dysfunction, pain and syringomyelia $(1,4,10)$.

Out of the symptomatic group the most frequently reported symptom was occipital headache, with certain distinguishing features - continuous headache with suboccipital localization with episodic retro-ocular component, almost never hemicranial and accompanied by vision disturbances, acoustic or vestibular disturbances, paresthesias and signs of medullary involvement. The pain is made worse by Valsalva type maneuvers, it is sometimes worse in the premenstrual period and it rarely is sensible to antidepressants, betablockers or triptans. It can be alleviated by topiramate, which lowers CSF pressure due to its effect as a carbonic anhidrase inhibitor $(5,11,12)$.

Neurologic examination is most often normal. Patients come seeking medical advice due to the worsening of headaches. Typical findings include vestibular-like disequilibrium and difficulty standing and walking in tandem.

Diagnosis of Chiari malformation type I is made using imaging studies, particularly MRI of the brain and cervical spine, which visualize the herniation of the cerebellar tonsils of at least $5 \mathrm{~mm}$ through the foramen magnum and into the central canal. Sagittal plane sequences are most useful in visualizing the posterior fossa and the occipitocervical junction. Several measurements need to be made: the diameter of the posterior fossa, the diameter of the foramen magnum (Twining line) and the size of the occipital bone. All these structures should be diminished in size in Chiari I malformations, otherwise a different cause for the herniation should be sought out. It is useful to mention that the foramen magnum is enlarged in tethered cord syndrome and Chiari type II and stenosed in Chiari type I, craniosynostosis, and achondroplasia. The sagittal and coronal planes are useful in visualizing the crowding of the posterior fossa and the foramen 
magnum and the downward herniation of the peglike tonsils through the foramen magnum. When available, dynamic MRI (cine-MRI) can be used to visualize the quality of CSF flow around the craniocervical junction $(3,11,13-15)$.

Differential diagnosis for Chiari malformations should include other causes of cerebellar herniation such as increased CSF pressure, hydrocephalus, space occupying lesions, CSF leaks, prolonged lombo-peritoneal shunting and low lying tonsils $(5,16)$.

Spinal cord dysfunction (syringomyelia) signs are the most frequent, followed by brainstem compression signs and cerebellum signs. Downbeat nystagmus is rarely found, even in cases of severe tonsilar herniation. Other possible, but rare findings include sinus bradycardia, syncope, pyramidal tract signs such as weakness, spasticity, hyperreflexia and Babinski responses $(5,17)$.

Spinal cord lesions such as hydromyelia, syringomyelia and/or syrigobulbia usually accompany Chiari malformations. Hydromyelia is an abnormal dilation within the central spinal canal in which there is excess cerebrospinal fluid. The excess fluid can damage the surrounding white matter fibers forming a cystic cavity called a syrinx; when this happens in the spinal cord the term used is syringomyelia, if the syrinx is located or extended at the medullary level, it is called syringobulbia.

Syringomyelia is a rare condition, but it is frequently found accompanying Chiari malformations. Most patients with Chiari malformations also have syringomyelia and in almost all patients with syringomyelia it is due to a Chiari malformation. Symptoms, when present, usually appear during adolescence or early adulthood.

Diagnosis of syringomyelia is based on imagery findings. MRI of the spine is the gold standard and it requires contrast enhancement in order to rule out spinal tumors. The MRI shows fluid-filled cavities within the spinal cord. Most frequent localization is at the cervical level; frequently there will be bony anomalies at the upper cervical level. MRI of the cervical spine will also visualize the Chiari malformation. If MRI cannot be performed, a myelography with post-myelographic-CT can be useful in establishing a diagnosis. The differential diagnosis includes amyotrophic lateral sclerosis, multiple sclerosis, spinal cord tumors, transverse myelitis and vitamin $\mathrm{B}_{12}$ deficiency $(15,18)$.

The management of Chiari malformations depends on the degree of severity and clinical signifi- cance. Asymptomatic patients without hydrocephalus or syrinx can be managed conservatively with MRI and clinical follow-up at 6 months. If the patient is still without symptoms and the MRI is not significantly changed, frequency of follow-up should be yearly $(19,20)$. However, some authors disagree with this approach and advocate for surgery in order to prevent further herniation and development of syrinx (21).

In paucisymptomatic patients with syrinx, but without changes in neurological examination, the management is controversial. There have been reports of spontaneous resolution of both tonsil herniation and syringomyelia (19). In these patients, conservative medical treatment can be attempted with carbonic anhidrase inhibitors to lower CSF pressure (15).

Pregnant patients with Chiari I malformations should undergo induced delivery and should avoid epidural or spinal anesthesia. If syringomyelia is associated, maneuvers that generate intrathoracic and intraabdominal pressure should be avoided, including vaginal delivery. (5)

Surgery is required in significantly symptomatic patients with symptoms of medullary compression, syringomyelia, myelopathy, cerebellar symptoms, severe neck pain or occipital headache, or in cases in which hydrocephalus is present. When hydrocephalus is present, it usually requires the placement of a ventriculo-peritoneal shunt. If there is a significant pressure upon the medulla, the best option is trans-oral resection of the compressing structure. When syrinx is present or when the patient is symptomatic without hydrocephalus, surgical conduit will consist of cranial cervical decompression with removal of the posterior arch of atlas and dural augmentation graft. The goal of surgical treatment is to allow CSF flow from the posterior fossa into the cervical subarachnoid space $(5,22,23)$.

\section{CONCLUSIONS}

Chiari malformations are not as rare as we think. They are probably more frequent but are left undiagnosed, due to lack of symptomatology. These patients have to be carefully selected before a decision for surgical treatment is made. Goals for surgery are brainstem decompression and reestablishment of normal CSF flow from the "crowded" posterior fossa and into the cervical subarachnoid space. 


\section{REFERENCES}

1. Sarnat H.B. Disorders of segmentation of the neural tube: Chiari malformations. Handbook of clinical neurology 2008; 87: 89-103.

2. Iskandar BJ O.W. Chiari malformations. Principles and practice of pediatric neurosurgery: Thieme; 1999: 165

3. Shane Tubbs JAP R., Jerry Oakes W. Chiari malformations. Youmans Neurological Surgery: Introduction to neurological surgery: W.B. Saunders; 2004.

4. Schijman E. History, anatomic forms, and pathogenesis of Chiari I malformations. Childs Nerv Syst 2004; 20(5): 323-8.

5. Daroff R.B., Bradley W.G. Bradley's neurology in clinical practice / [edited by] Robert B. Daroff ... [et al.]. 6th ed. Philadelphia, PA: Elsevier/Saunders; 2012

6. Milhorat T.H., Nishikawa M., Kula R.W., Dlugacz Y.D. Mechanisms of cerebellar tonsil herniation in patients with Chiari malformations as guide to clinical management. Acta Neurochir (Wien) 2010; 152(7): 1117-27.

7. Speer M.C., Enterline D.S., Mehltretter L., et al. Review Article: Chiari Type I Malformation with or Without Syringomyelia: Prevalence and Genetics. Journal of genetic counseling 2003; 12(4): 297-311.

8. Bloch S., Van Rensburg M.J., Danziger J. The Arnold-Chiari malformation. Clinical radiology 1974; 25(3): 335-41.

9. Elster A.D., Chen M.Y. Chiari I malformations: clinical and radiologic reappraisal. Radiology 1992; 183(2): 347-53.

10. Goh S., Bottrell C.L., Aiken A.H., Dillon W.P., Wu Y.W. Presyrinx in children with Chiari malformations. Neurology 2008; 71(5): 351-6.

11. Meadows J., Kraut M., Guarnieri M., Haroun R.I., Carson B.S. Asymptomatic Chiari Type I malformations identified on magnetic resonance imaging. J Neurosurg 2000; 92(6): 920-6.

12. Milhorat T.H., Chou M.W., Trinidad E.M., et al. Chiari I malformation redefined: clinical and radiographic findings for 364 symptomatic patients. Neurosurgery 1999; 44(5): 1005-17.

13. Caldarelli M., Di Rocco C. Diagnosis of Chiari I malformation and related syringomyelia: radiological and neurophysiological studies. Childs Nerv Syst 2004; 20(5): 332-5.
14. Gundry C.R., Heithoff K.B. Imaging evaluation of patients with spinal deformity. The Orthopedic clinics of North America 1994; 25(2): 247-64.

15. Najem C.E. Syringomyelia. Ferri's Clinical Advisor 2016: Elsevier; 2016: 1187e3-e4.

16. Fenton D.S. Chiari type I malformation. imaging painful spine disorders: Saunders; 2011: 108-13.

17. Steinbok P. Clinical features of Chiari I malformations. Childs Nerv Syst 2004; 20(5): 329-31.

18. Michael Devereaux D.H. Bradley's Neurology in Clinical Practice. Disorders of Bones, Joints, Ligaments, and Meninges: Elsevier; 2016: 1736-65.e2.

19. Novegno F., Caldarelli M., Massa A., et al. The natural history of the Chiari Type I anomaly. Journal of neurosurgery Pediatrics 2008; 2(3): 179-87.

20. Schijman E., Steinbok P. International survey on the management of Chiari I malformation and syringomyelia. Childs Nerv Syst 2004; 20(5): 341-8.

21. Navarro R., Olavarria G., Seshadri R., Gonzales-Portillo G., McLone D.G., Tomita T. Surgical results of posterior fossa decompression for patients with Chiari I malformation. Childs Nerv Syst 2004; 20(5): 349-56.

22. Alden T.D., Ojemann J.G., Park T.S. Surgical treatment of Chiari I malformation: indications and approaches. Neurosurgical focus 2001; 11(1): E2.

23. Haroun R.I., Guarnieri M., Meadow J.J., Kraut M., Carson B.S. Current opinions for the treatment of syringomyelia and chiari malformations: survey of the Pediatric Section of the American Association of Neurological Surgeons. Pediatric neurosurgery 2000; 33(6): 311-7. 